\title{
High quality epitaxial Znse and the relationship between electron mobility and photoluminescence characteristics
}

\author{
Konstantinos P. Giapis, Da-Cheng Lu, and Klavs F. Jensen \\ Department of Chemical Engineering and Materials Science, University of Minnesota, Minneapolis, \\ Minnesota 55455
}

(Received 11 August 1988; accepted for publication 10 November 1988)

\begin{abstract}
High quality epitaxial hayers of nominally undoped ZnSe have been grown by metalorganic chemical vapor deposition at low temperature $\left(325^{\circ} \mathrm{C}\right.$ ) and pressure ( 30 Torr), using dimethylzinc and hydrogen selenide. All layers were unintentionally doped $n$ type with net carrier concentrations of $6.4 \times 10^{14}-1.5 \times 10^{16} \mathrm{~cm}^{-3}$ and exhibited very high mobility at room temperature (up to $500 \mathrm{~cm}^{2} / \mathrm{N}$ ) as well as at $77 \mathrm{~K}$, where the measured value of $9250 \mathrm{~cm}^{2} /$ $V \mathrm{~s}$ is the highest so far reported for vapor phase growth. Additional evidence for the high quality of the material is provided by photoluminescence. Experimental results indicate a correlation between the photoluminescence characteristics and the electrical properties that may be useful in assessing the quaity of ZnSe films.
\end{abstract}

High quality epitaxial layers of ZnSe that can be doped controllably have a well-recognized potential use in bive light-emitting diodes and lasers. Significant progress towards this goal has recently been made by metalorganic chemical vapor deposition (MOCVD), inchding improvements in surface morphology, near-band-edge (NBE) photoluminescence, and electrical properties, ${ }^{1-3}$ as well as reports of measurable p-type conductivity in $\mathrm{ZnSe}{ }^{4}$ Here, we report further improvements in material quality of nominally undoped $Z$ nSe prepared by MOCVD, as evidenced by its photoluminescence (PL) characteristics and its electrical properties. Experimental data indicate an empirical correlation between electron mobility and $\mathrm{PL}$ characteristics, which is proposed as a means for assessing high quality $\mathrm{ZnSe}$ films, independent of the growth technique.

ZnSe was grown in a loadlock equipped MOCVD reactor, designed for the growth of $\mathrm{ZnSe}$. The reactants used were "electronic" grade dimethylzinc (DMZ) and hydrogen selenide ( $\mathrm{H}_{2} \mathrm{Se}$ ), in situ produced from Pd-purified hydrogen and $99.9999 \%$ pure selenim. The $\mathrm{H}_{2} \mathrm{Se}$ was formed in a small reactor unit, separated from selenium and partial. ly condensed, so that a controlled amount of $\mathrm{H}_{2} \mathrm{Se}$ in $\mathrm{H}_{2}$ could be introduced into the reaction chamber. Typically, fow rates of DMZ and $\mathrm{H}_{2}$ Se were kept at 20 and $35 \mathrm{kmol}$ min, respectively. The substrates were semi-insulating GaAs (100), oriented $2^{\circ}$ towards $\langle 10\rangle$ and were prepared according to standard procedures. ${ }^{5}$ The GaAs wafer was placed on a Mo susceptor inside the loadlock and pumped down to a pressure of $1 \times 10^{-7}$ Torr, then transferred into the main reactor chamber where it was heat treated at $650^{\circ} \mathrm{C}$ for $10 \mathrm{~min}$ in $1 \mathrm{slm} \mathrm{H}_{2}$ flow, while the reactor was kept at 300 Torr. The samples discussed here were grown at $325^{\circ} \mathrm{C}$, while the reactor pressure was varied between 30-100 Torr and the nozzle to susceptor distance between $20-50 \mathrm{~mm}$. This led to a variation in the growth rate between 24 and 32 $\mu m / h$ and had a profound effect on the incorporation of impurities in the films.

All layers investigated in the present study were single crystalline, of thickness between $8-32 \mu \mathrm{m}$ and showed features common to ZnSe of comparable thickness grown from identical sources, i.e., hillocks in the $\langle 110\rangle$ direction. ${ }^{5,6}$ Electrical properties were evaluated by measurements of resistivity and Hall effect. Ohmic contacts were made by pressing small pieces of In on the freshly grown $Z$ ZnSe surface, followed by annealing in purited $\mathrm{H}_{2}$ for $5 \mathrm{~min}$ at $300^{\circ} \mathrm{C}$. Formation of ohmic contacts was verified using a curve tracer. Photoluminescence spectra recorded before and after annealing were identical in sharpness and intensity of peaks, indicating that annealing at the above conditions had no significant efiect on the properties of the firms.

Results of flm characteristics at 300 and at $77 \mathrm{~K}$ are listed in Table 1 . All samples investigated were $n$ type with $300 \mathrm{~K}$ net carrier concentrations ranging from $6.4 \times 10^{14}$ to $1.5 \times 10^{16} \mathrm{~cm}^{-3}$. The $77 \mathrm{~K}$ value of mobility for run No. $62 \mathrm{of}$

TABLE I. Experimental and calculated electrical properties of undoped ZnSe. $n$ and $\mu$ are the net carrier concentration and mobility at the corresponding temperature. $N_{A}$ and $N_{D}$ are the total aceptor and donor concentrations, $\theta$ is the compensation ratio. $\Delta E\left(I_{x}\right)$ is the linewidth of the $I_{x}$ peak in the PL spectrat and $R\left(I_{x} / \mathrm{DL}\right)$ is the maximum NBE peak to the DE intensity ratio, commonly cited in the hiterature.

\begin{tabular}{|c|c|c|c|c|c|c|c|c|c|c|}
\hline Rurs No. & $\begin{array}{c}\text { Thickness } \\
\text { (jum) }\end{array}$ & $\left(10^{15} \mathrm{~cm}^{n_{300}}\right)$ & $\begin{array}{c}n_{7 ?} \\
\left(10^{15} \mathrm{~cm}^{3}\right)\end{array}$ & $\begin{array}{c}\mu_{300} \\
\left(\mathrm{~cm}^{2} / \mathrm{s}\right)\end{array}$ & $\begin{array}{c}\mu_{7 \gamma} \\
\left(\mathrm{cm}^{2} / \mathrm{Vs}\right)\end{array}$ & $\begin{array}{c}N_{A} \\
\left(10^{15} \mathrm{~cm}^{-3}\right)\end{array}$ & $\begin{array}{c}N_{D} \\
\left(10^{15} \mathrm{~cm}^{-3}\right)\end{array}$ & $\theta$ & $\begin{array}{l}\Delta E\left(t_{x}\right) \\
\text { (meV) }\end{array}$ & $R\left(i_{z} / \mathrm{DL}\right)$ \\
\hline 48 & 32.0 & 15.0 & 7,0 & 500 & 3250 & 13.0 & 20.0 & 0.65 & 1.8 & 5000 \\
\hline 50 & 31.2 & 4.8 & 2.5 & 460 & 5600 & 6.4 & 8.9 & 0.72 & 1.2 & 500 \\
\hline 51 & 31.8 & 10.0 & 4.1 & 480 & 4600 & 8.3 & 12.0 & 0.67 & 1.6 & 1900 \\
\hline 54 & 12.4 & 2.6 & 1.3 & 450 & 5650 & 6.1 & 7.4 & 0.82 & 1.5 & 1000 \\
\hline 55 & 15.0 & 2.1 & 1.2 & 475 & 7700 & 4.3 & 5.5 & 0.78 & 1.0 & 500 \\
\hline 62 & 8.3 & 0.9 & 0.6 & 475 & 9250 & 3.3 & 4.0 & 0.84 & 1.1 & 200 \\
\hline 68 & $\$ 1.0$ & 2.6 & 1.4 & 460 & 6250 & 5.5 & 6.9 & 0.79 & 1.1 & 270 \\
\hline
\end{tabular}


$9250 \mathrm{~cm}^{2} / \mathrm{V}$ s is to our knowledge the highest so far reported for ZnSe grown by a vapor phase deposition technique, including molecular beam epitaxy (MBE). Similar values have been reported for bulk-grown material. ${ }^{7}$ In Figs. 1(a) and $\mathrm{i}(\mathrm{b})$ we show the total electron mobility versus carrier concentration at 300 and $77 \mathrm{~K}$, respectively. Data from other MOCVD ${ }^{1-3}$ and $\mathrm{MBE}^{8}$ studies of nominally undoped $\mathrm{ZnSe}$ are included for comparison. The solid curves have been reproduced from the theoretical work on electron transport in ZnSe by Ruda. ${ }^{9}$ The $300 \mathrm{~K}$ mobility data, close to a constant (average) value of $480 \mathrm{~cm}^{2} / \mathrm{V}$ s, fall outside the theoretical predictions of electron transport in $\mathrm{ZnSS}$ at room temperature. However, the model appears to work well at 77 $\mathrm{K}$, where ionized impurity scattering becomes the dominant mechanism limiting electron mobility. For this scattering process, the mobility is given by the Brooks-Herring formu$\mathrm{la}^{10}{ }^{10}$ which is used to estimate $N_{A}, N_{D}$, and the compensation ratio $\theta$ from experimental values of the $77 \mathrm{~K}$ mobility and net carrier concentration. The results are summarized in Table I. For our fims, $\theta$ falls between 0.65 and 0.85 in good agreement with the more extensive calculations by $R$ uda, ${ }^{9}$ as can be seen by the fitting of our points from the curve with $\theta=0.7$. The close agreement between our measured $77 \mathrm{~K}$ mobilities and theoretically predicted inherent limit mobiiitees is a further indication of the very high purity and quality of our material.

There is a possibility of measuring anomalousiy high apparent mobilities, resulting from inadvertently introducing conducting inhonogeneities in our ZnSe films. ${ }^{11}$ However, this is unlikely because: (1) No mobilities exceeding the theoretical latice scattering limited values have been measured. (2) ZnSe films with $77 \mathrm{~K}$ mobility in the high
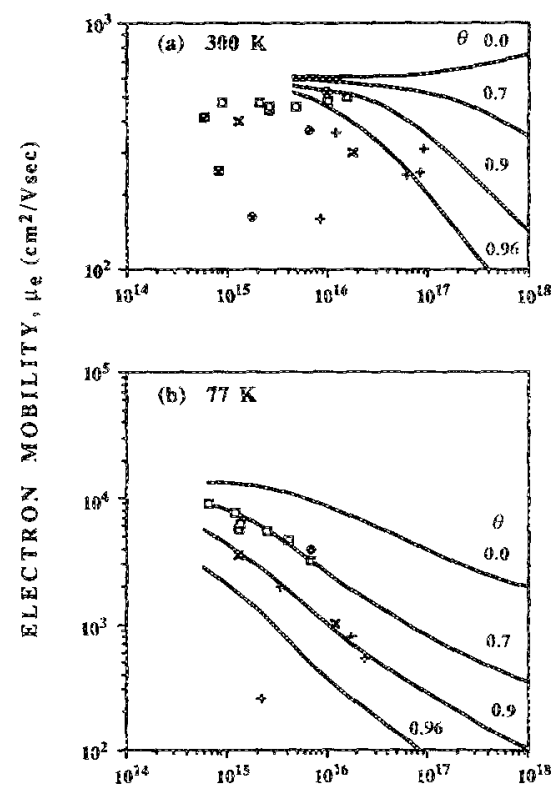

CARRIER CONCENTRATRON, $\left.\operatorname{tm}^{-3}\right)$

FIG. 1. Comparison of the Hall electron mobility vs carrier concentration at (a) $300 \mathrm{~K}$ and (b) $77 \mathrm{~K}$ for $Z \mathbf{Z n S e}$. The symbols correspond to expcrimental data points for nominally undoped fims as follows: ( $\square$ ) this study, $(+)$ Ref. 1, (X) Ref. 2, (Ref. 3. (Nef. 8. The solid curves represent the calculated values of mobility vs carrier concentration for different compensation ratios, reproduced from the work of Ruda (Ref. 9 ).
$8000 \mathrm{~cm}^{2} / \mathrm{V}$ s region have been grown reproducibly. (3) If the samples had conducting inhomogeneities, they would appear to be quite uncompensated, ${ }^{18}$ but run No. 62 (with the highest mobility at $77 \mathrm{~K}$ ) is the most compensated among the runs. (4) The variation of the apparent Hali constant with the magnetic field has been considered for several samples. Upon increasing the magnetic field the Hall constant decreased as expected from theory. ${ }^{12}$

Photoluminescence (PL) spectra at room temperature and $9 \mathrm{~K}$ were obtained by using the UV output of an $\mathrm{Ar}^{+}$ion laser at low power densities $\left(<30 \mathrm{~mW} / \mathrm{cm}^{2}\right)$ in an unfocused beam of diameter $2 \mathrm{~mm}$, and analyzed using a $0.85-\mathrm{m}$ SPEX $: 403$ double monochromator and photon counting electronics. Spectral resolution in the range of interest was better than $0.5 \mathrm{~cm}^{-1}$. The $\mathrm{PL}$ spectra at room temperature and $9 \mathrm{~K}$ of our highest mobility sample are shown in Figs. 2 (a) and 2 (b). This sample was grown at $325^{\circ} \mathrm{C}, 30$ Torr, and $[\mathrm{VI} / \mathrm{II}]$ ratio of 1.75 . Both spectra are composed of a strong near-band-edge (NBE) emission, while the broad emission at smaller energies (deep levels) is very weak. The $9 \mathrm{~K}$ spectrum [Fig. 2(b)] is dominated by an intense and narrow peak at $2.7954 \mathrm{eV}$, identified as the commonly observed $I_{x} \cdot{ }^{13}$ This may be assigned to $I_{3} \cdot \mathrm{Cl}$, described by Dean et al. ${ }^{i 4}$ which would explain the $n$-type conductivity. In the expansion of the NBE emission spectra [Fig. 2(b), inset], a second dominant peak is clearly defined at $\mathbf{2 . 7 9 7 0}$
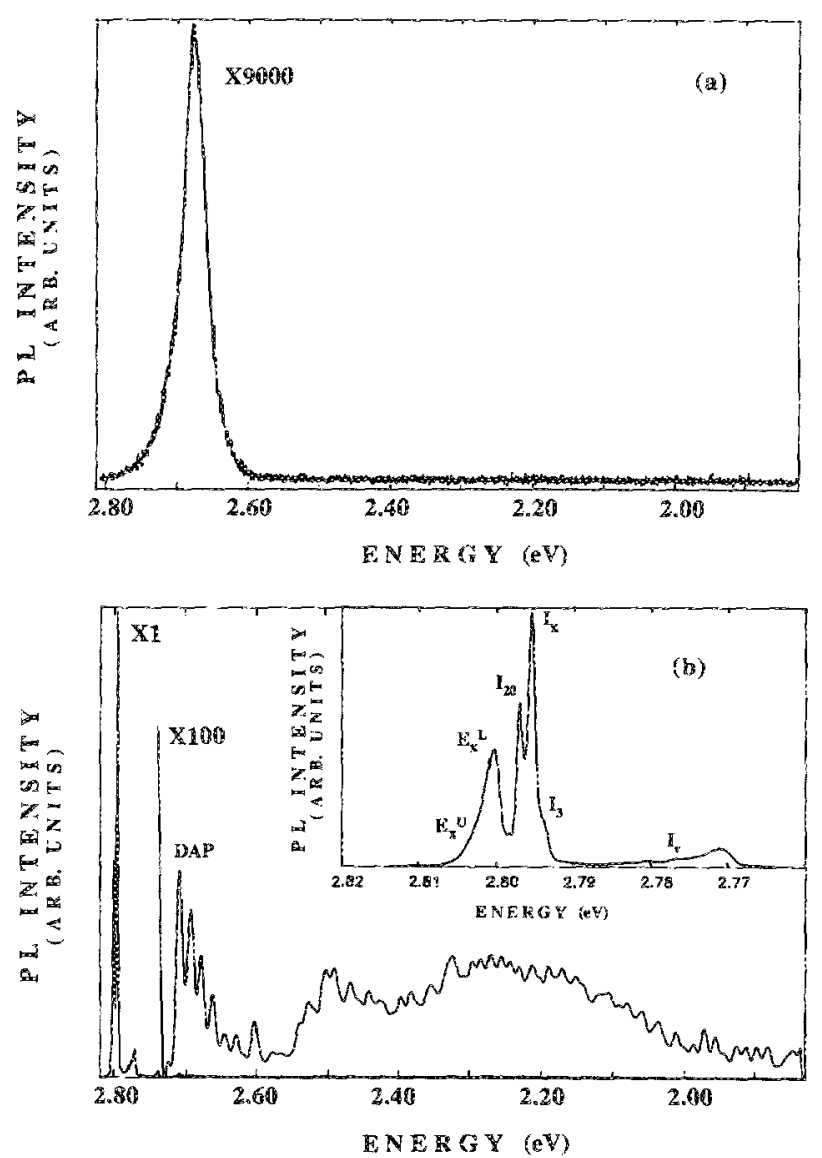

FIG. 2. (a) Room-temperature photoluminescence spectrum for the $\mathrm{ZnSe}$ sample with the highest mobility at $77 \mathrm{~K}$. (b) The corresponding $9 \mathrm{~K} \mathrm{PL}$ spectrum. The inset of this figure is an enlargement of the NBE luminescence. The dominant feature in this spectrum is $I_{x}$, but the $E_{x}^{L}$ is distinct and of comparable intensity with $I_{x}$. 
$\mathrm{eV}$; this is referred to as $I_{20}$ and has been attributed to recombination of excitons bound to shallow extrinsic neutral donors. ${ }^{15}$ There are also two peaks due to free-exciton recombination: the very intense lower branch polariton, denoted $E_{x}^{L}$ at $2.8002 \mathrm{eV}$ and, on its high-energy side, the upper branch polariton, $E_{x}^{U}$ at $2.8026 \mathrm{eV}$. The peak on the lower energy side of the $I_{x}$ at $2.7938 \mathrm{eV}$ is the $I_{3}$ line, presumably due to excitons bound to an ionized donor ${ }^{13}$ One also distinguishes

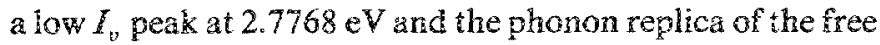
exciton at $2.7711 \mathrm{eV}$. Very weak donor-acceptor pair emission is also observed, which means that the concentration of acceptor-like impurities or defects is considerably reduced in the firms discussed here." As compared to other $\mathrm{L}$ results in the literature, $3,13,15,16$ our PL spectra are distinguished for their sharp, narrow, and distinct peaks in the NBE region and the weakness of the $I_{1}^{d}$ hine, usually appearing at 2.782 $\mathrm{eV}$ and being attributed to $\mathrm{Zn}$ vacancies. 3,15 In fact, since there is no discemible $I d i$ line in the PL spectra of all the samples discussed in this letter, we believe that our material is very close to being stoichiometric. Another manifestation of the purity of our fims comes from the two to three orders of magnitude smaller deep level (DL) emission ( $h v<2.5$ $\mathrm{eV}$ ), as compared to the NBE dominant peak. The DL emission appears as a weak, broad $C u$-green emission at approximately $2.3 \mathrm{eV}$.

Features of low-temperature PL spectra, including the wid th of the $I_{x}$ peak $(\triangle E)$, the ratio of the dominant NBE to $D L$ intensities ( $R$ value), and the intensity of the dominant NBE emission, are frequently used in the literature ${ }^{16}$ to judge the relative quality of the 2 nSe films. Linewidths, measured at half maximum, serve as a criterion of hlm quality, since the PL lines broaden as the density of impurities and the sample inhomogeneity increase. ${ }^{6,17}$ The $I_{x}$ of Fig. 2(b) has a linewidth of $\triangle E=1.1 \mathrm{eV}$, among the narrowest of our samples with measurable mobility. The $R$ value of the same sample was approximately 200 , but we have grown material with an $R$ value as high as 5000 . However, our results (see Table 1 ) indicate a reverse relationship between the $R$ value and the quaity of the material as determined by the electrical properties, in contradiction with previous reports., ${ }^{3,6}$ This can be explained as follows: a very intense NBE peak, usually appearing with a large linewidth (broadening), is related to a higher concentration of radiative electron recombination centers in the films. ${ }^{17}$ In the particular case of the dominant donor bound excitonic emission $l_{x}$, this inplies a higher concentration of donors, which will result in an increased electron screening and scattering and therefore lower mobility values, especialiy at low temperatures where the ionized impurity scattering is dominant. This trend is clear in 7 . where the $77 \mathrm{~K}$ mobility is shown to decrease with the linewidth of the $I_{x}$ peak as well as with the intensity ratio $\left[I_{x} / E_{x}^{L}\right]$. This coupling of the electrical properties with the characteristics may provide a better means of assessing the quality of undoped $\mathrm{ZnSe}$, rather than just interpreting the ambiguous $R$ value, which may aiso vary with both the excitation power and wavelength. ${ }^{16}$ Therefore, in cases of highly resistive undoped $Z$ nSe films, one could use $\mathrm{PL}$ spectra with a dominant $\mathrm{NBE}$ emission (i.e., $R$ value 275) to judge the quality by examining the linewidth of the

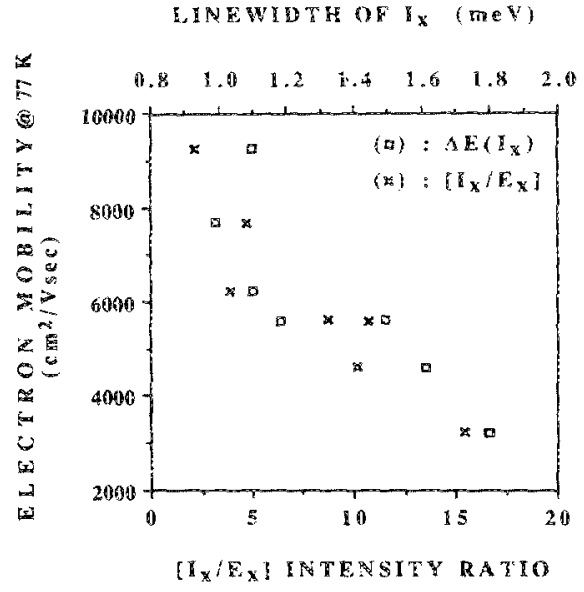

FIG. 3. Electron mobility at $77 \mathrm{~K}$ plotted vs the $\mathrm{PL}$ intensity of the $I_{x}$ peak nomalized over the intensity of the $E_{x}$ peak $(X)$, as well as vs the linewidth of the $I_{x}$ peak ( $\square$ ).

dominant peak as well as its intensity ratio over the intensity of the free exciton.

In summary, we have shown that by using low-temperature MOCVD with additional purifation of the starting materials, it is possible to grow epitaxial ZnSe with higher mobility than previously reported. We also have provided experimental resuits showing a direct relationship between the slectrical properties and the PL characteristics of high quality $Z$ ZnSe, which may be useful as a means for assessing the quality of $Z$ nSe films. This study indicates that $n$-type (and possibly $p$-rype) conductivity in $Z$ nSe is limited by extrinsic impurities rather than vacancies or self-interstitials, which renews the hope of obtaining efficient $p-n$ junction light-emitting devices from $Z$ ZnSe.

The authors are grateful to $\mathbb{J}$. E. Pots, $\mathrm{H}$. Cheng, and $\mathrm{I}$. M. DePuydt for discussions and encouragement conceming this project. We also wish to thank $G$. Haugen for technical assistance with the PL measurements. This work was supported by $3 \mathrm{M}, \mathrm{NSF}$ (PY), and the Dreyfus Foundation.

'S. Fujita, F.Yodo, and A. Sasaki, \$. Cryst. Growth 72, 27 (1985).

${ }^{2}$ A. Yoshikawa, S. Yamaga, K. Tanaka, and H. Kasa, J. Cryst. Growth 72, 13 (1985); Jpn. I. Appl. Phys. 23, L424 (1984).

${ }^{3}$ G. Fan. J. Davies, M. Parrot, and J. Winiams, J. Electron. Mater. 15, 25, (1986).

'T. Yasuda, I. Mitsuishi, and $\mathrm{H}$. Kukimoto, Appl. Phys. Lett. 52, 57 (1988).

5W. Stutius, J. Electron. Mater. 10,95 (1981).

'S. Fujita, T. Yodo, Y. Matsuda, and A. Sasaki, J. Cryst. Growth 71, 169 (1985).

${ }^{7}$ M. Aven, 5. Appí. Phys. 42, 1204 (1971).

${ }^{8}$ T. Yao, J. Cryst. Growth 72, $31(1985)$; T. Yao and Y. Okada, Jpr. I. Appl. Phys. 25, 82!(1986).

${ }^{9}$ H. Ruda, I. Appl. Phys. 59, 1220 (1986)

10H. Brooks, in Advances in Electronics and Electron Physics, edited by $\mathrm{L}$. Marton (Academic, New York, 1955), p. 158.

${ }^{1} \mathrm{C}$. Wolfe, G. Stilman, and J. Rossi, J. Electrochem, Soc. 19, 250 (1972).

${ }^{12} \mathrm{G}$. Stillman and C. Wolfe, Thin Solid Fims 31, 69 (1976).

${ }^{13}$ T. Yao, Y. Makita, and S. Maekawa, Jpn. J. Appl. Phys. 20, L741 (1981).

${ }^{14}$ P. J. Dean, D. C. Herbert, C. J. Werkhoven, B. J. Fitzpatrick, and R. N. Bhargava, Bhys. Rev. B 23, 4888 (1981).

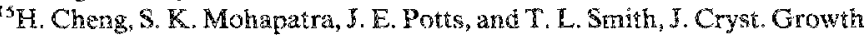
$81,512(1987)$

10J. M. DePuydt, H. Chen, J. E. Potts, T. L, Smith, and S. K. Mohapatra, J. Appl. Phys. $62,4756(1987)$

${ }^{17}$ G. B. Stringrellow and R. Linnebach, J. Appl. Bhys. $\$$. 2212 (1980). 